\title{
A Facile Synthesis of a Polyhydroxylated 2-
}

\section{Azabicyclo[3.2.1]octane.}

\author{
Damon D. Reed and Stephen C. Bergmeier*
}

Department of Chemistry and Biochemistry, Ohio University, Athens, Ohio 45701

\section{bergmeis@ohiou.edu}

Contents

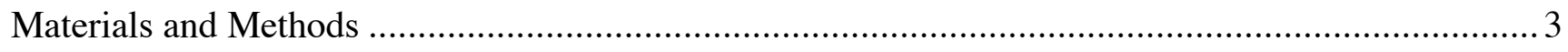

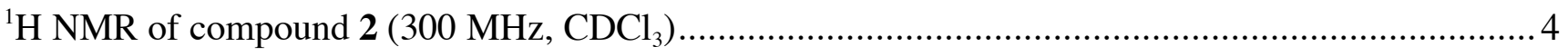

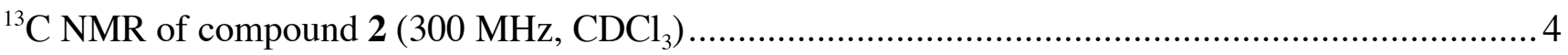

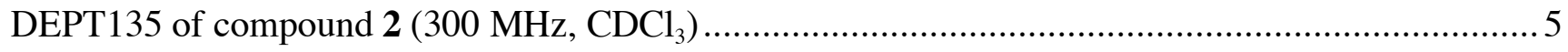

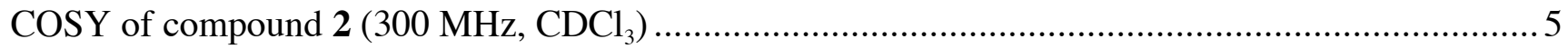

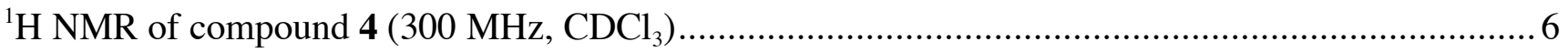

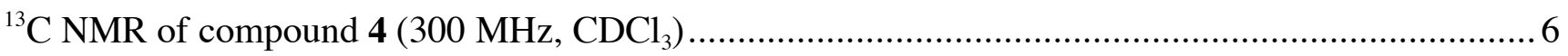

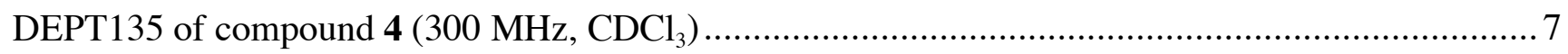

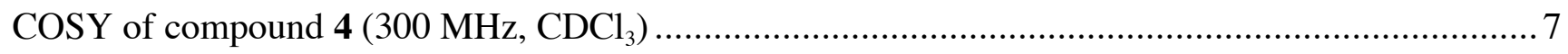

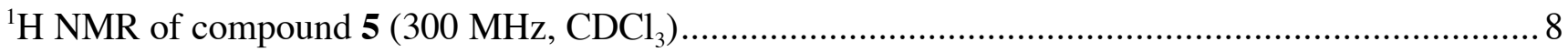

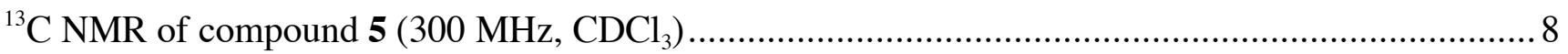

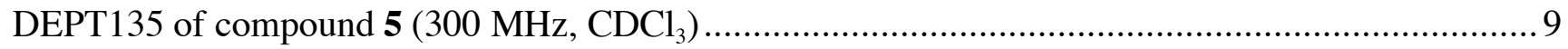

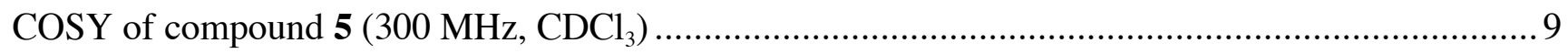

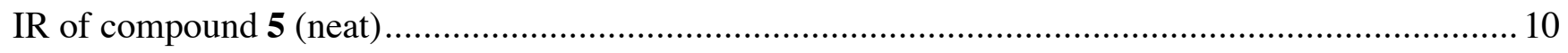

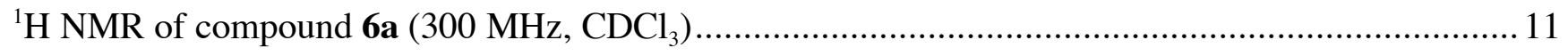

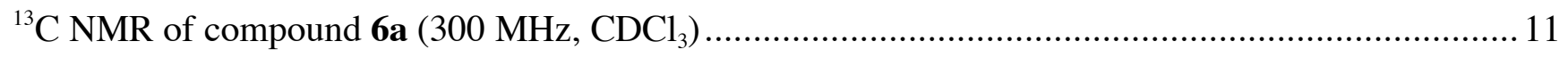

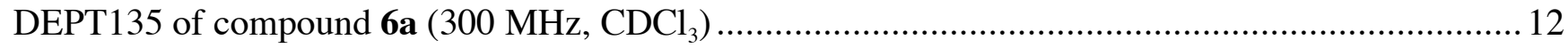




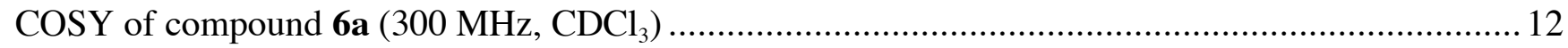

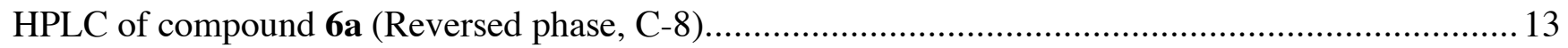

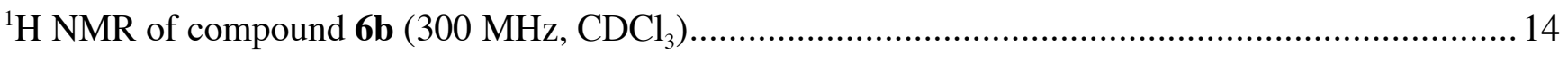

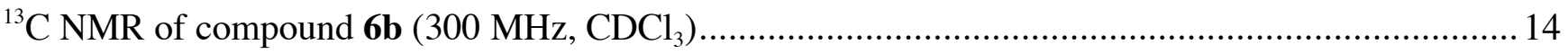

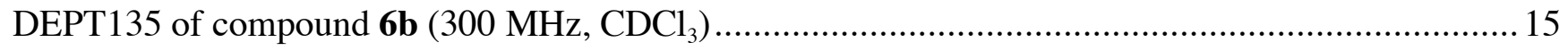

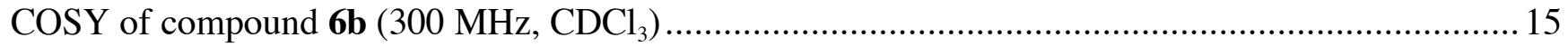

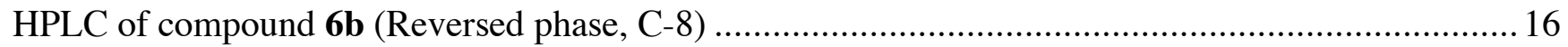

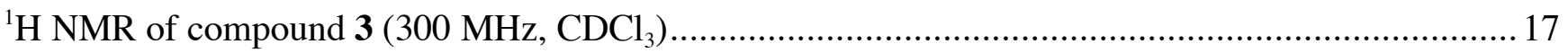

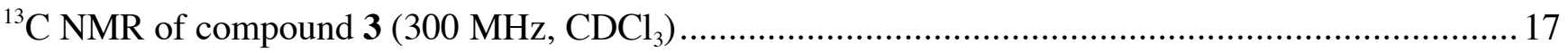

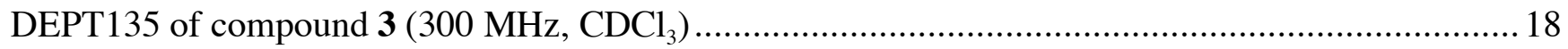

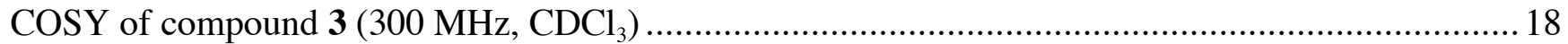




\section{Materials and Methods}

All chemicals were purchased from the usual commercial suppliers and used without further purification unless noted. THF was dried using a column purification system. IRs were obtained neat. HPLCs were obtained on a C8 column $(15 \mathrm{~cm} \mathrm{x} 4.6 \mathrm{~mm}, 5 \mu \mathrm{m})$ using a flow rate of $2.0 \mathrm{~mL} / \mathrm{min}$ with a gradient (95:5 to 0:100) of $\mathrm{H}_{2} \mathrm{O} / \mathrm{CH}_{3} \mathrm{CN}$ over 16 min. Melting points were obtained using a capillary melting point apparatus and are uncorrected. Photolysis was carried out through a pyrex filter with a high pressure Xe-Hg lamp (450-watt). $\mathrm{R}_{f}^{\prime}$ s were obtained by thin layer chromatography on aluminum backed, silica coated, TLC plates containing a fluorescent $(254 \mathrm{~nm})$ indicator. Spots were identified via UV or staining with ninhydrin/ethanol mixture followed by heating. Compounds were purified by either an automated flash purification system utilizing silica gel or Biotage KP-NH cartridges or by flash

chromatography using 32-63D $60 \AA$ silica gel according to the method of Still. ${ }^{1}$ NMRs were obtained at $300\left({ }^{1} \mathrm{H}\right)$ or $75 \mathrm{MHz}\left({ }^{13} \mathrm{C}\right)$. Nomenclature for NMR spectra is as follows: $\mathrm{s}=$ singlet, bs = broad singlet, $\mathrm{d}=$ doublet, $\mathrm{dd}=$ doublet of doublets, $\mathrm{t}=$ triplet, $\mathrm{m}=$ multiplet.

\footnotetext{
${ }^{1}$ Still, W. C.; Kahn, M.; Mitra, A. J. Org. Chem. 1978, 43, 2923.
} 
प1?

N-tosyl-2-azabicyclo[3.2.1]octa-3,6-diene (2)

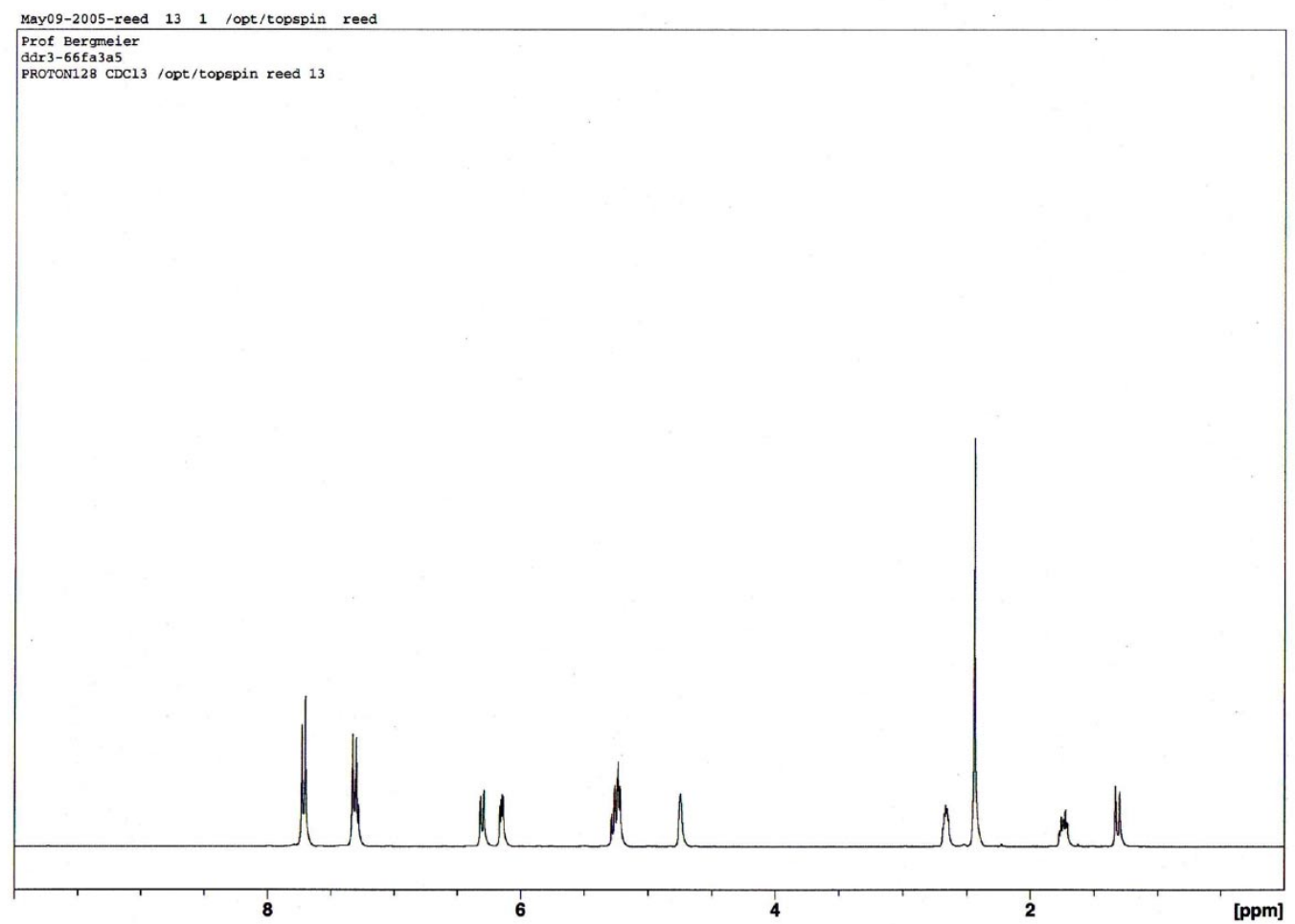

May09-2005-reed $11 \quad 1$ /opt/topspin reed

Prof Bergmeier

C13CPD CDC13 /opt/topspin reed 13

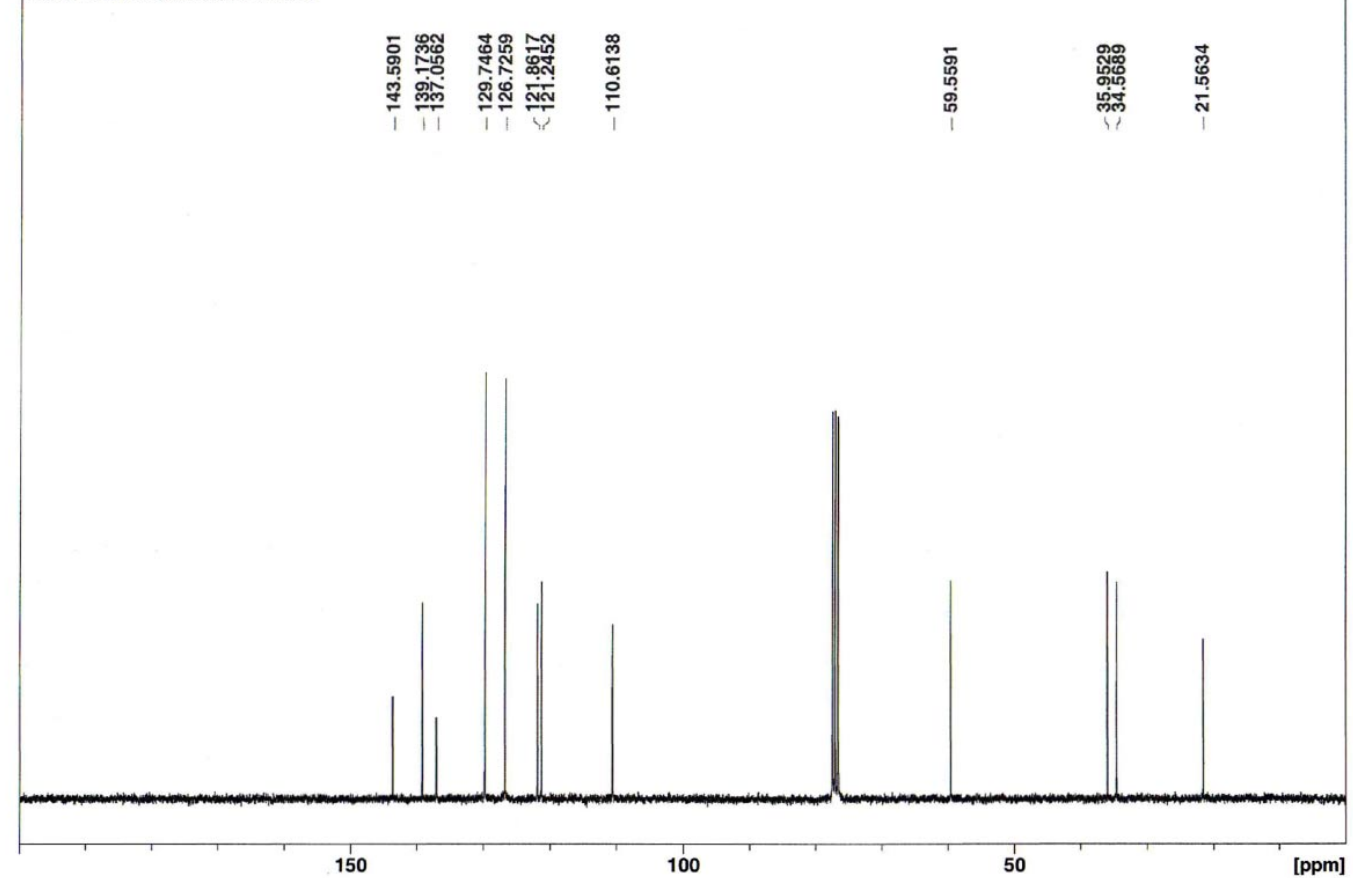


[1]

N-tosyl-2-azabicyclo[3.2.1]octa-3,6-diene (2)
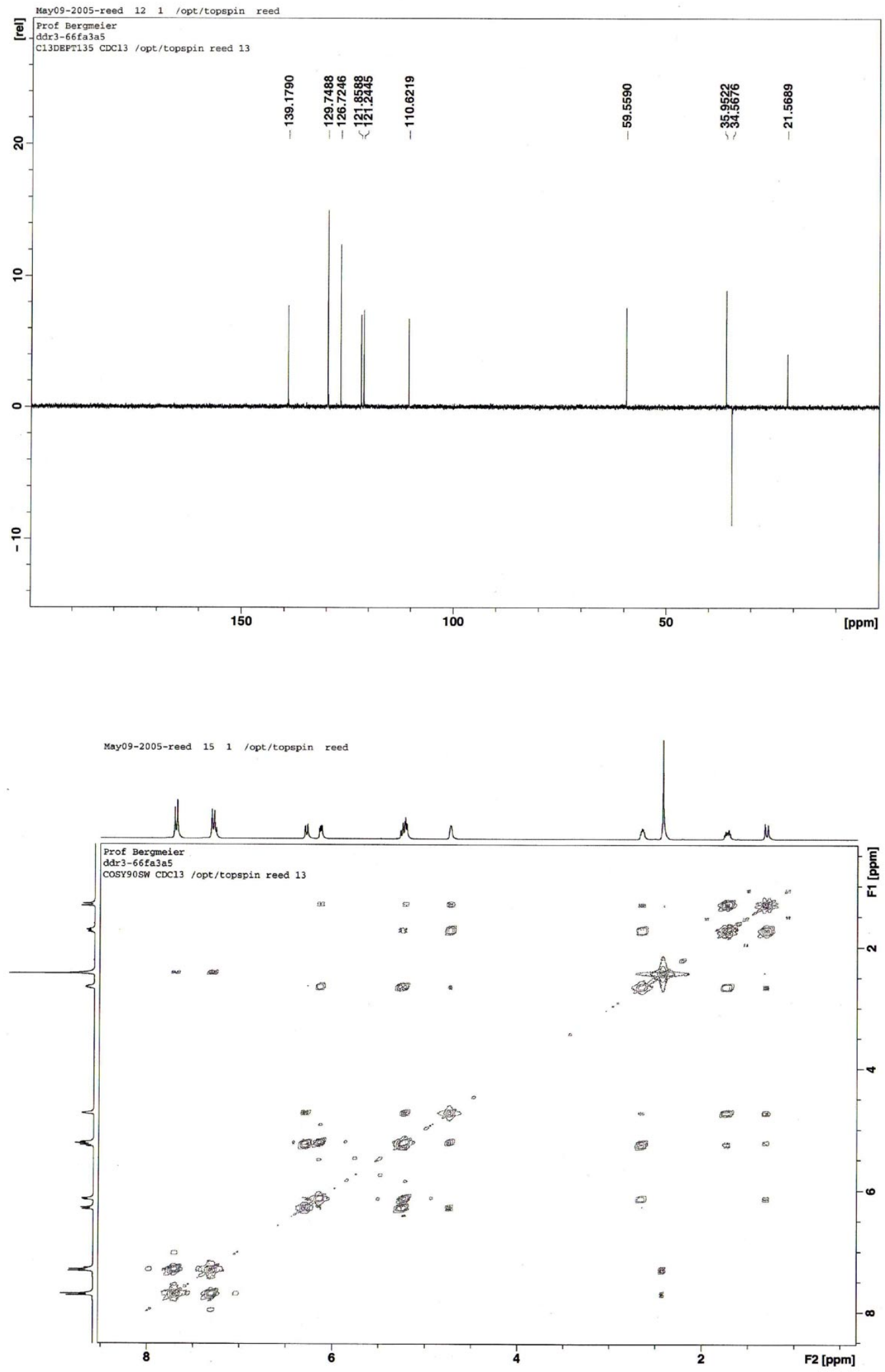
${ }_{\text {HO }}^{{ }_{\mathrm{T}}}$
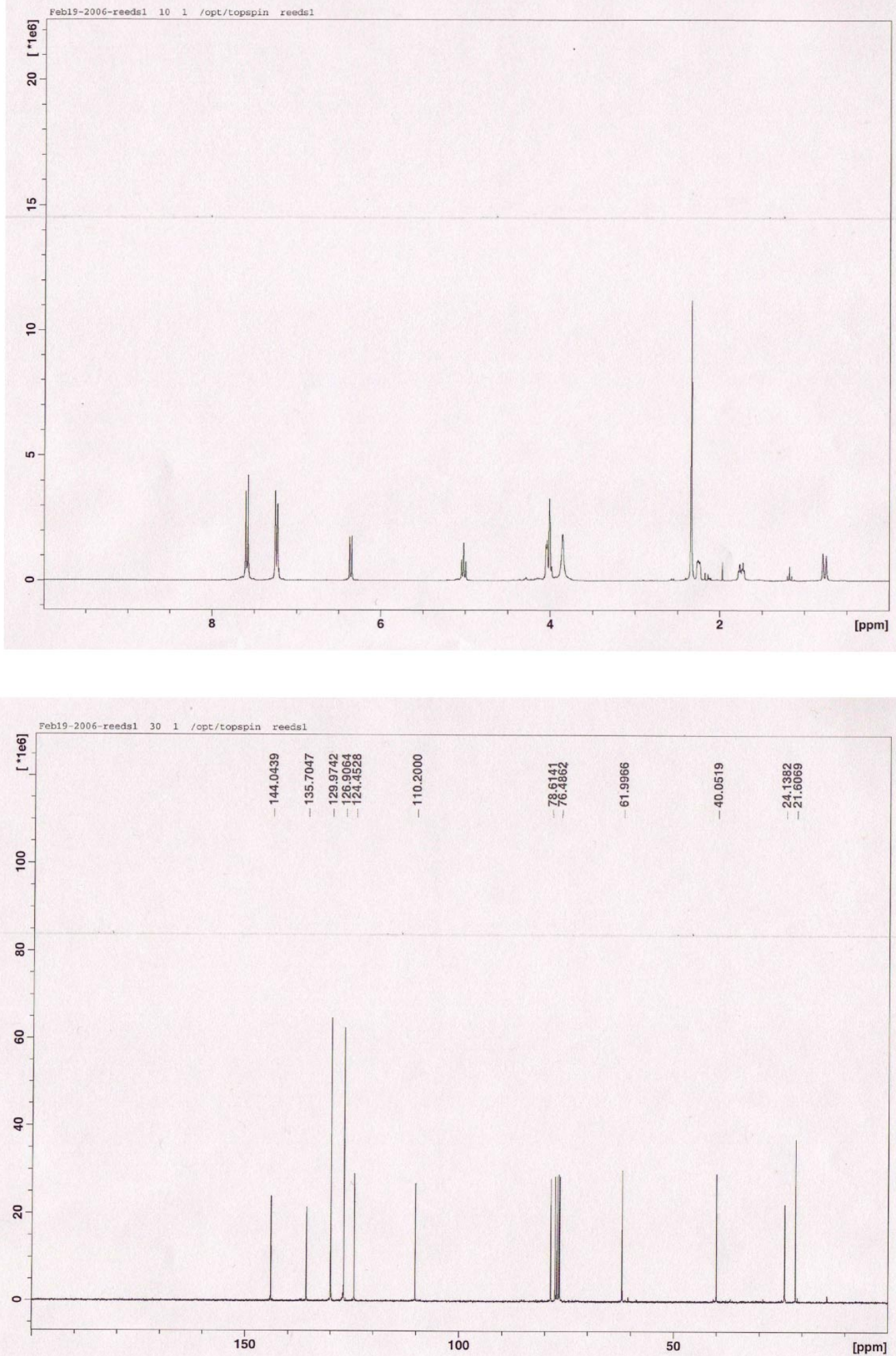
21

N-tosyl-2-aza-bicyclo[3.2.1]oct-3-ene-6,7-diol (4)
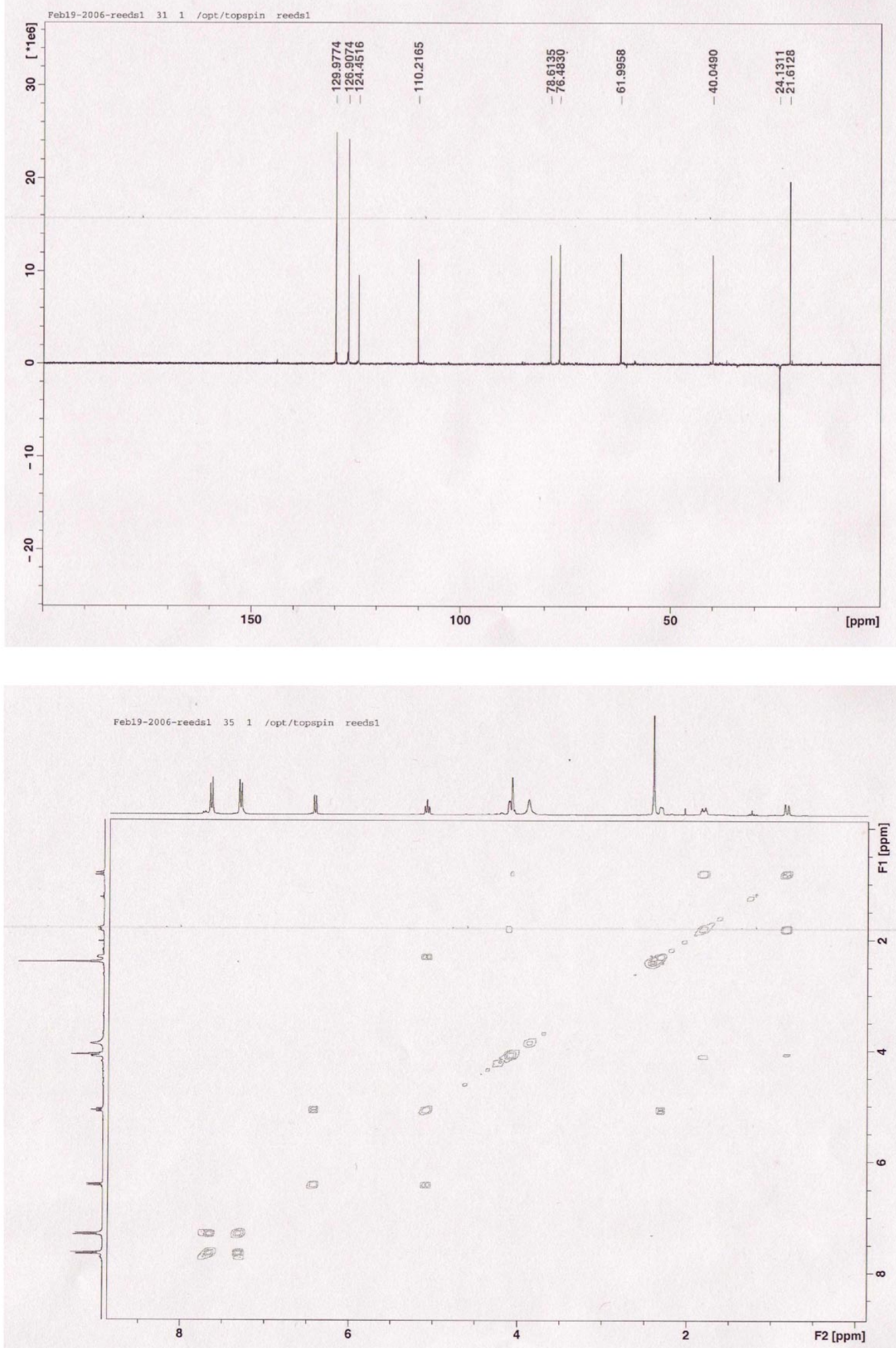
6,7-exo-isopropylidenedioxy-N-tosyl-2-aza-bicyclo[3.2.1]oct-3-ene (5)

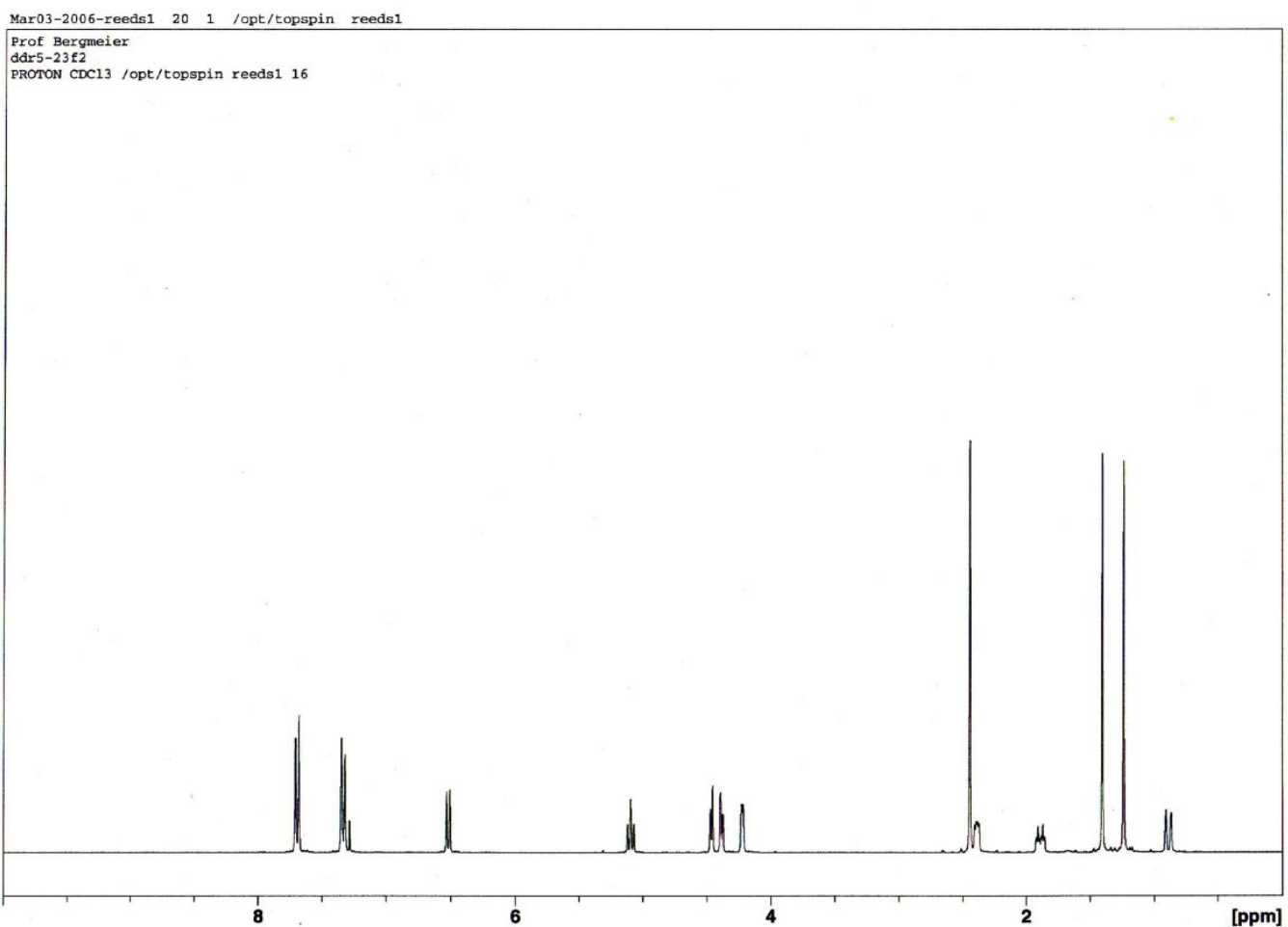

Mar02-2006-reeds1 $30 \quad 1$ /opt/topspin reeds1 Prof Bergmeier
dar $5-23 f 14-19$

disCPD CDC13 /opt/topspin reeds1 22

\begin{tabular}{|c|c|c|c|c|c|c|}
\hline $\begin{array}{l}\frac{m}{\infty} \\
\stackrel{\infty}{\infty} \\
\stackrel{m}{\tau}\end{array}$ & 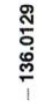 & 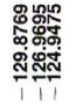 & 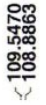 & 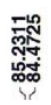 & 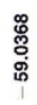 & 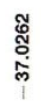 \\
\hline
\end{tabular}

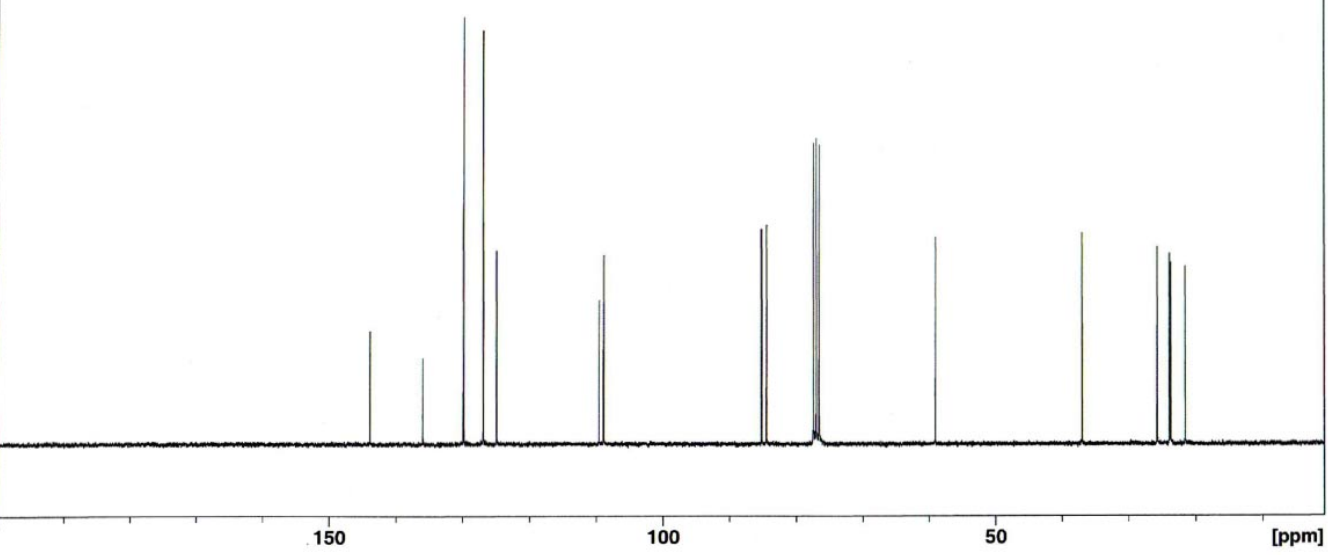




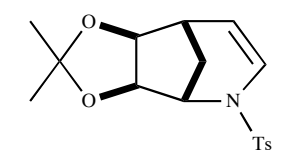

6,7-exo-isopropylidenedioxy-N-tosyl-2-aza-bicyclo[3.2.1]oct-3-ene (5)
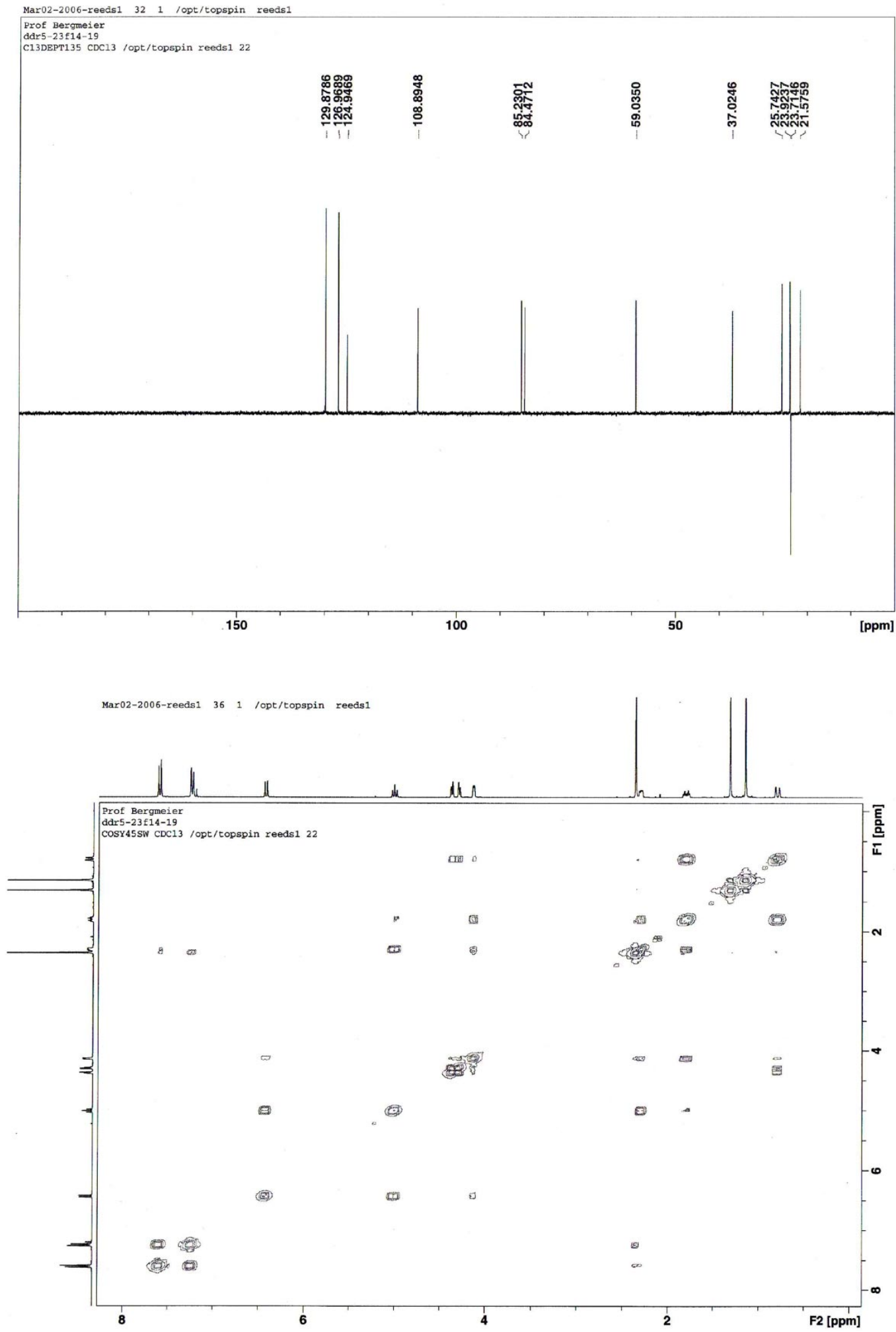


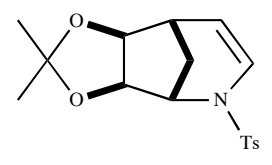

6,7-exo-isopropylidenedioxy-N-tosyl-2-aza-bicyclo[3.2.1]oct-3-ene (5)

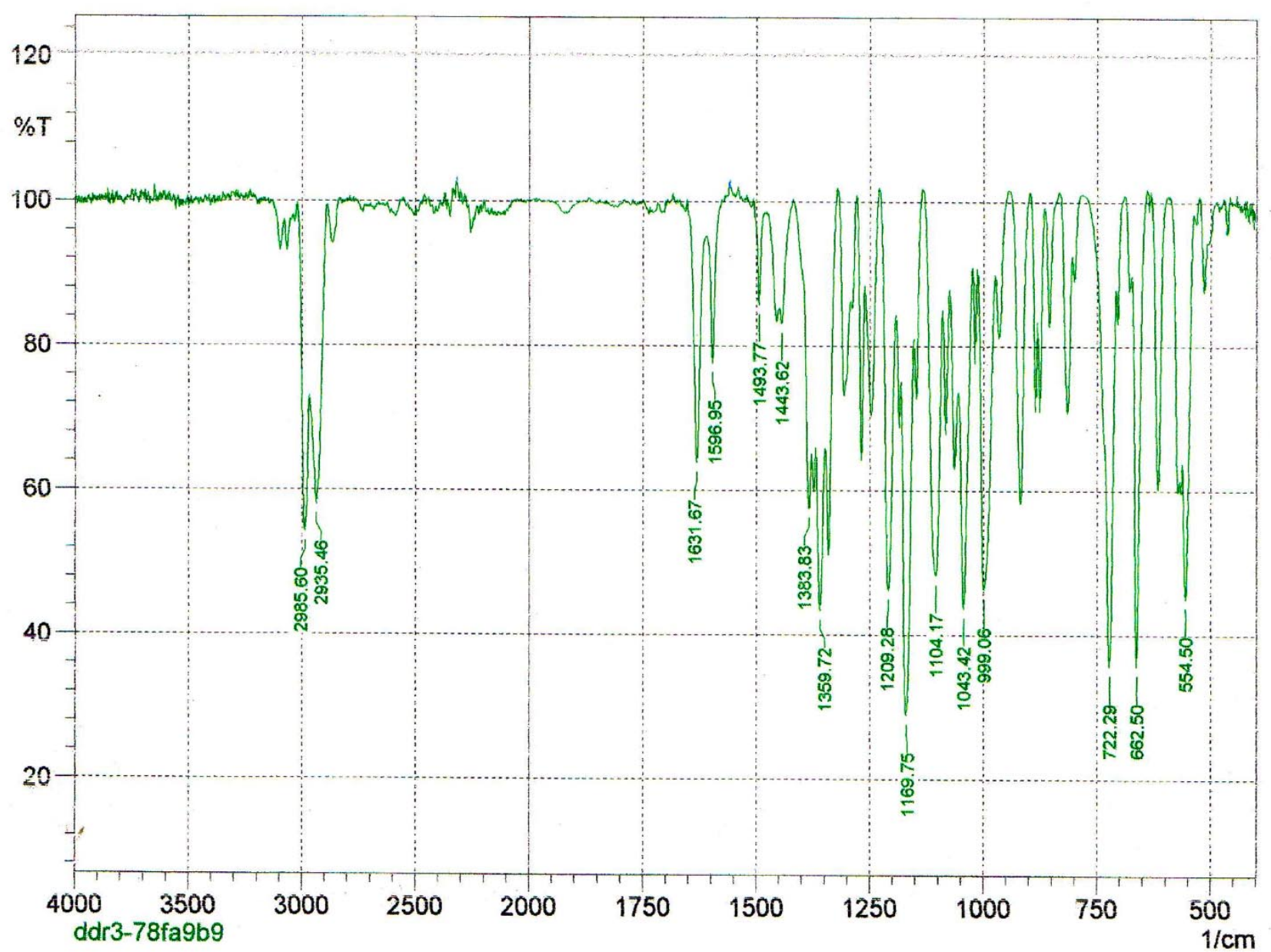

Date/Time; $03 / 14 / 200509: 32.45 \mathrm{AM}$

File Name: C:Program FilestShimadzuIPsolution!data

idamonldar3-78fa969.smt 


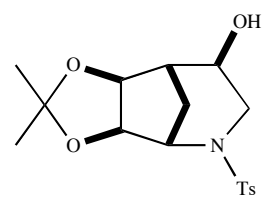

4-exo-hydroxy-6,7-exo-isopropylidenedioxy-N-tosyl-2-aza-bicyclo[3.2.1]octane (6a)

Feb14-2006-reeds1 $23 \quad 1$ /opt/topspin reeds1
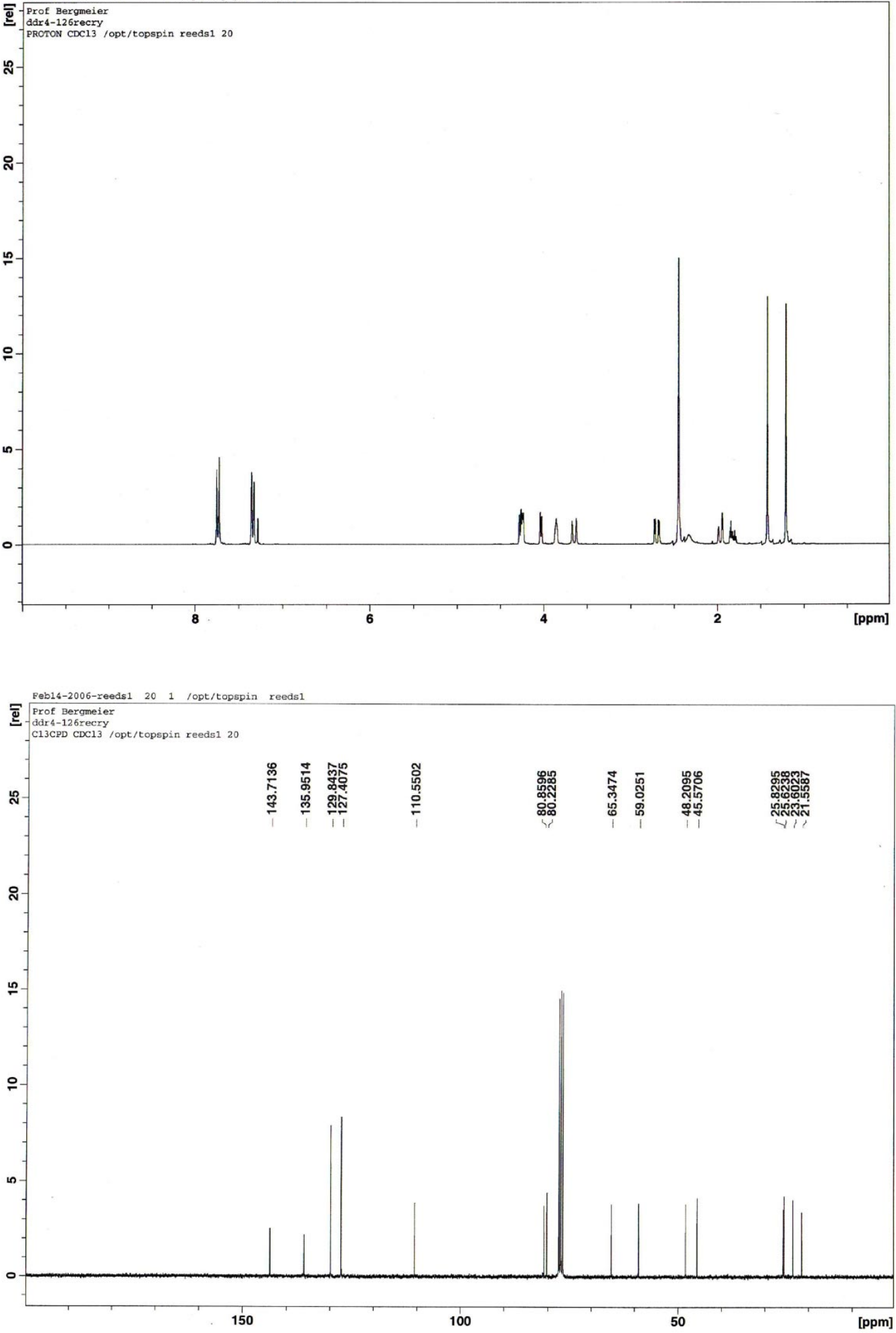


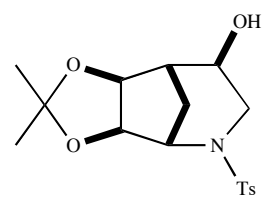

4-exo-hydroxy-6,7-exo-isopropylidenedioxy-N-tosyl-2-aza-bicyclo[3.2.1]octane (6a)
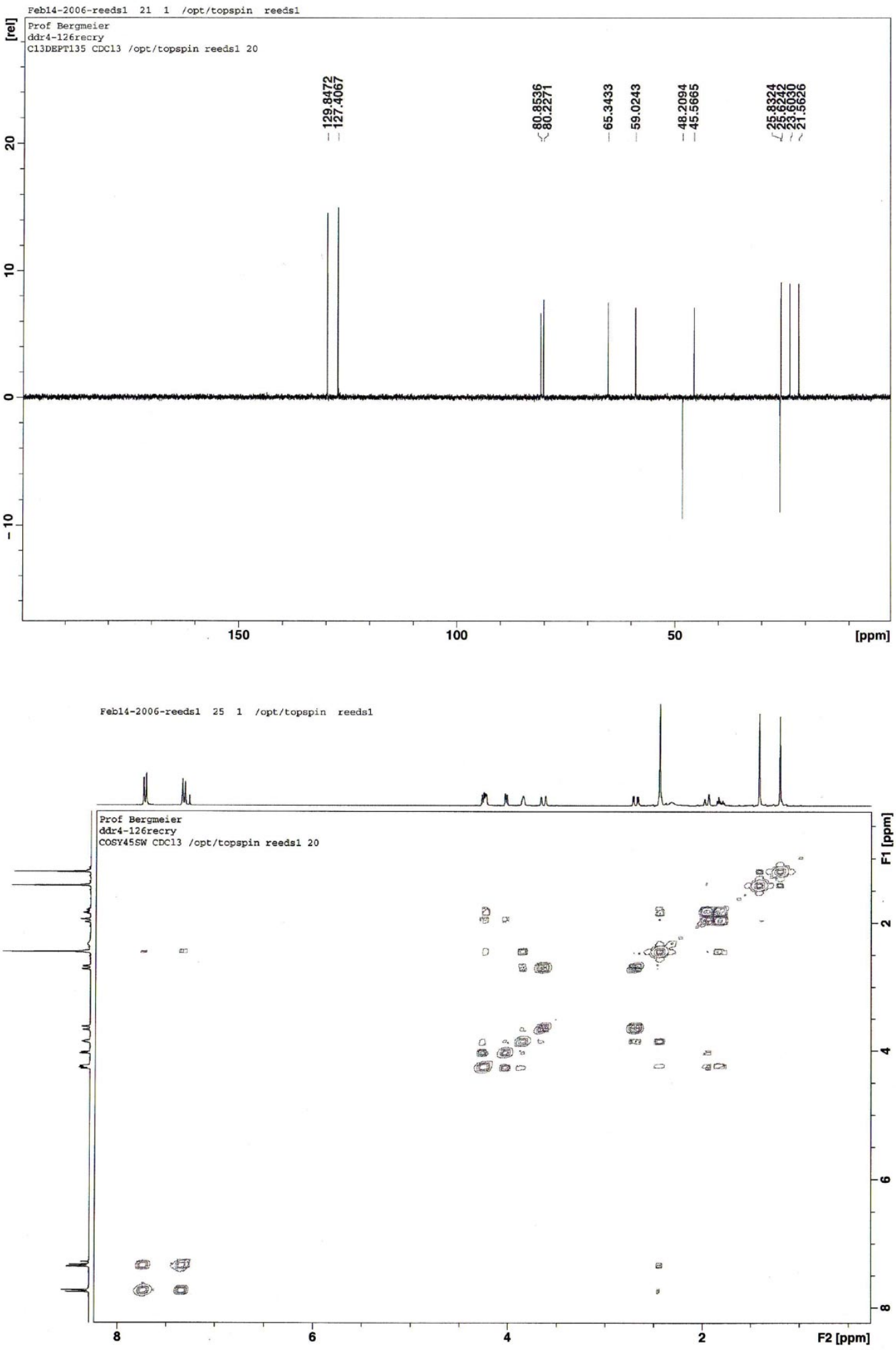


\section{Area \% Report}

Method Name: C: $\backslash$ CLASS-VP\Enterprise $\backslash$ Projects $\backslash$ Default $\backslash$ Method $\backslash$ damon $\backslash$ shut down.met

Data:

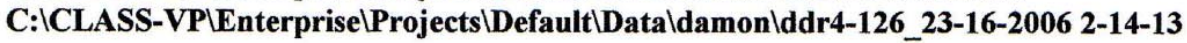

PM.dat

User: System

Acquired: $\quad 3 / 16 / 2006$ 2:15:04 PM

Printed: $\quad 3 / 17 / 2006$ 12:42:28 PM

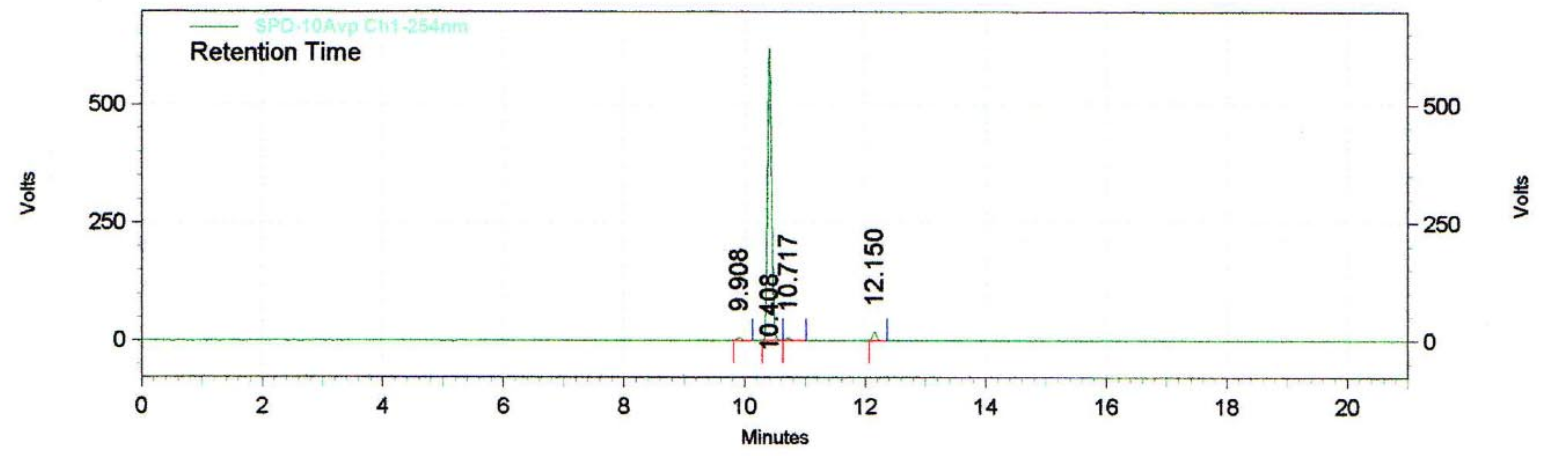

SPD-10Avp

Ch1-254nm

Results

Retention Time

9.908

10.408

10.717

12.150

Area

Area \%

0.97

95.25

0.94

2.84

28609

86742

SPD-10Avp

Ch2-276nm

Results

\begin{tabular}{|r|r|r|r|r|}
\hline \multicolumn{1}{r}{ Retention Time } & Area & Area \% & Height & Height \% \\
\hline 10.408 & 370327 & 100.00 & 79100 & 100.00 \\
\hline Totals & 370327 & 100.00 & 79100 & 100.00 \\
\hline
\end{tabular}



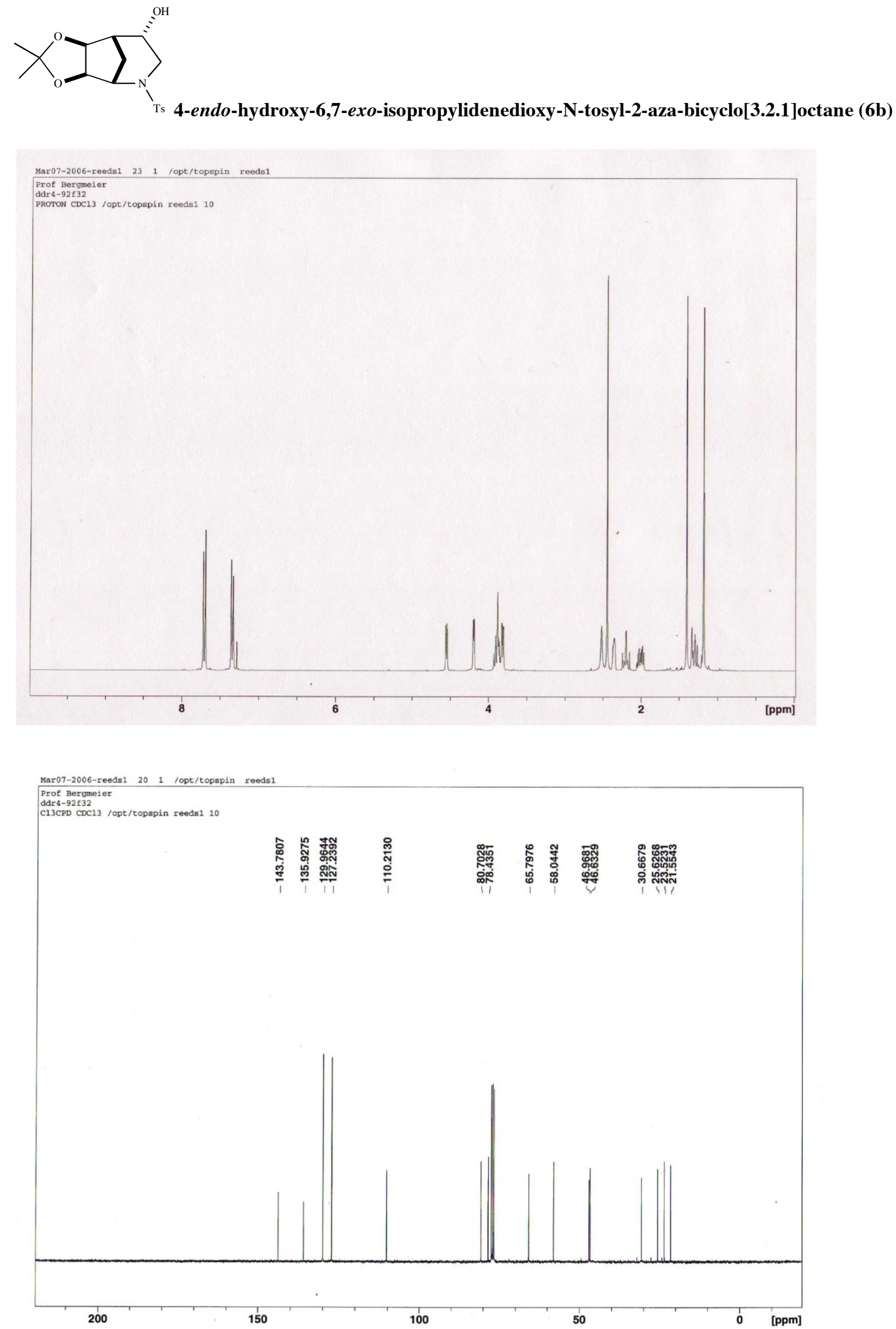

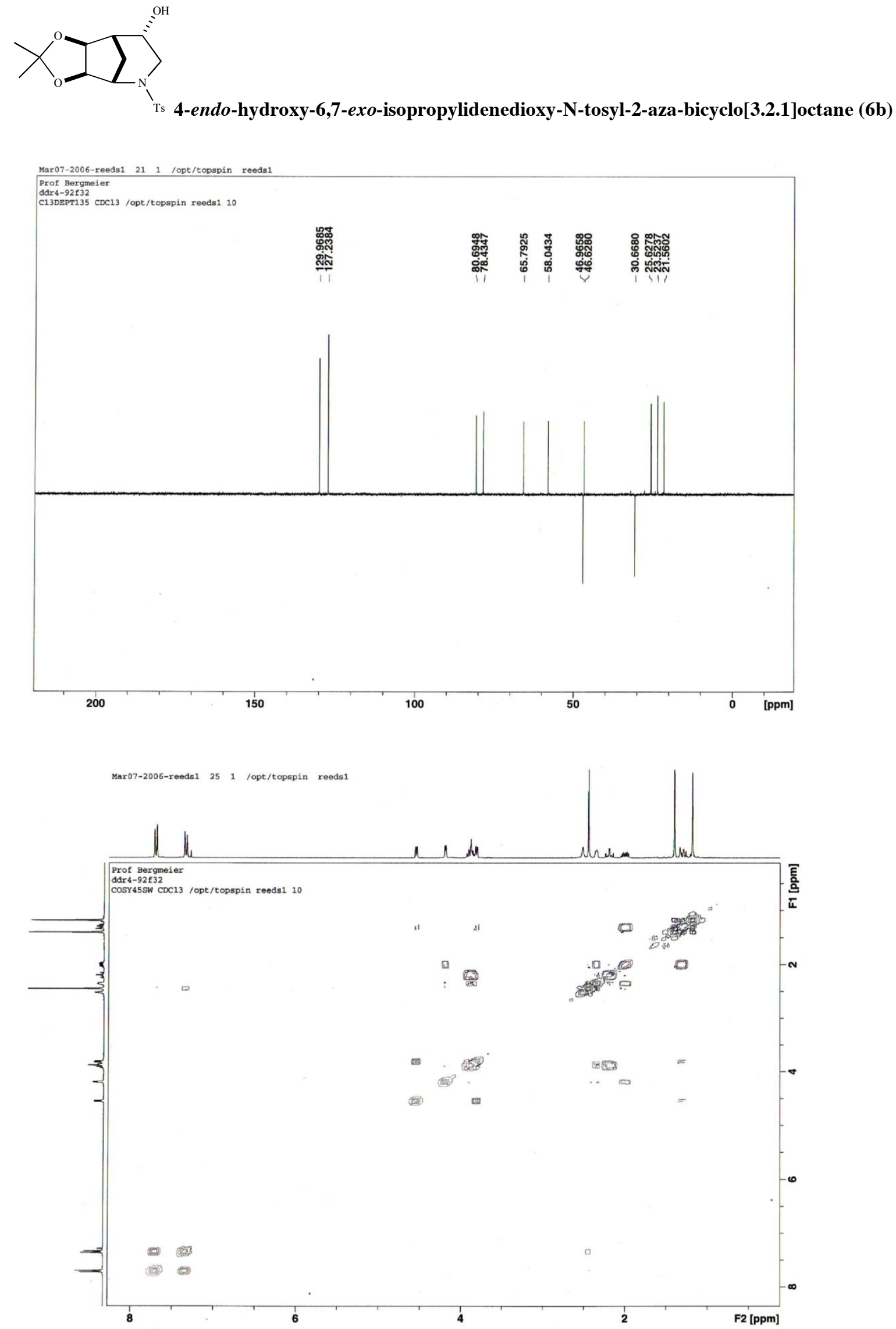


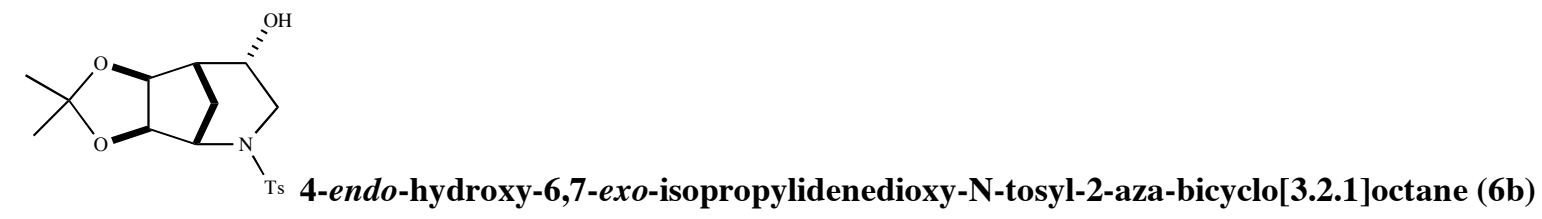

Area \% Report

Page 1 of 2

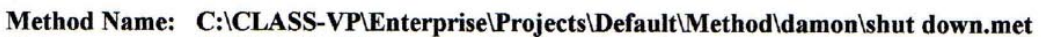

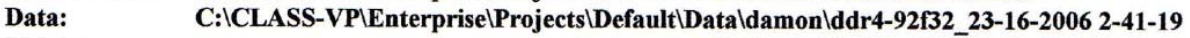

PM.dat

User: $\quad$ System

Acquired: $\quad 3 / 16 / 2006$ 2:42:11 PM

Printed: $\quad 3 / 17 / 2006$ 12:39:51 PM

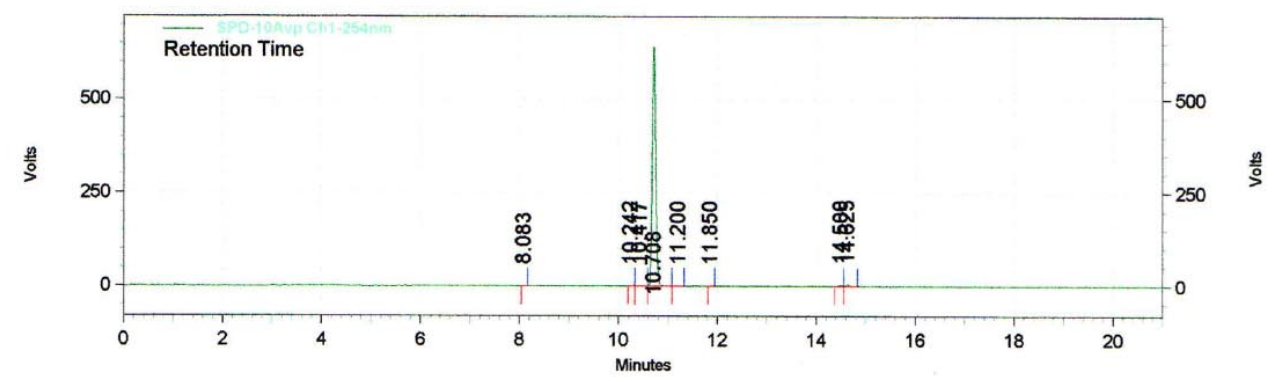

\section{SPD-10Avp \\ Ch1-254nm \\ Results}

Retention Time

8.083

10.242

10.417

10.708

11.200

11.850

14.500

14.625

\begin{tabular}{rrrr} 
Area & Area \% & Height & Height \% \\
\hline 811 & 0.03 & 233 & 0.04 \\
1673 & 0.05 & 427 & 0.07 \\
2474 & 0.08 & 535 & 0.08 \\
305295 & 98.28 & 641939 & 98.44 \\
5753 & 0.18 & 782 & 0.12 \\
1104 & 0.04 & 287 & 0.04 \\
18240 & 0.58 & 3195 & 0.49 \\
23984 & 0.76 & 4742 & 0.73
\end{tabular}

\begin{tabular}{|r|r|r|r|r|}
\hline Totals & 3149334 & 100.00 & 652140 & 100.00 \\
\hline
\end{tabular}

SPD-10Avp Ch2-276nm

Results

\begin{tabular}{|rrr|r|r|}
$\begin{array}{r}\text { Retention Time } \\
\text { Area }\end{array}$ & Area \% & Height & Height \% \\
\hline 10.708 & 400804 & 100.00 & 83502 & 100.00 \\
\hline Totals & 400804 & 100.00 & 83502 & 100.00 \\
\hline
\end{tabular}


4-exo-hydroxy-6,7-exo-isopropylidenedioxy-N-aza-bicyclo[3.2.1]octane (3)

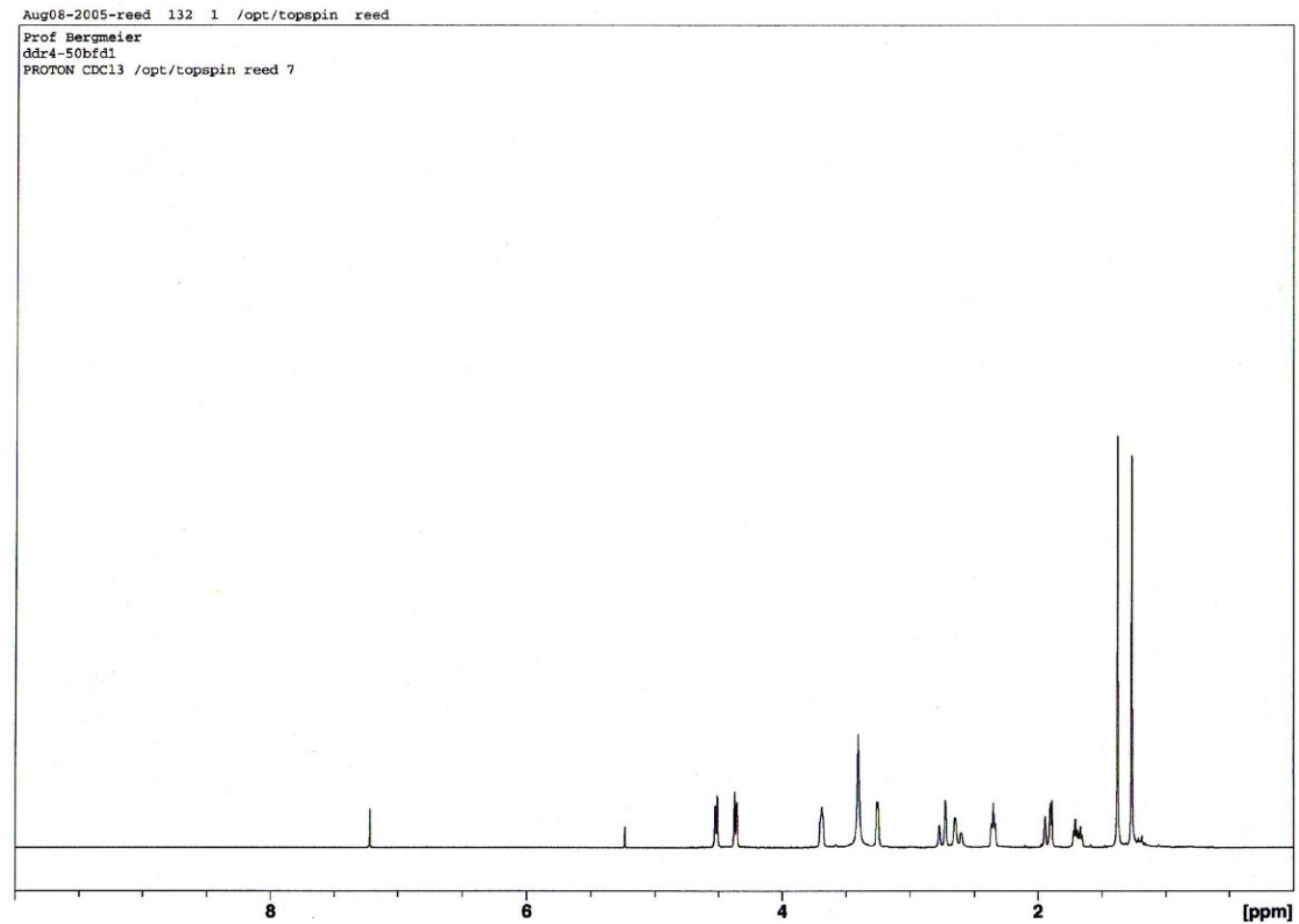

Aug08-2005-reed 1301 /opt/topspin reed

Prof Bergmeier

dar4-50bfd1
C13CPD CDC13 /ODt/topspin reed 7

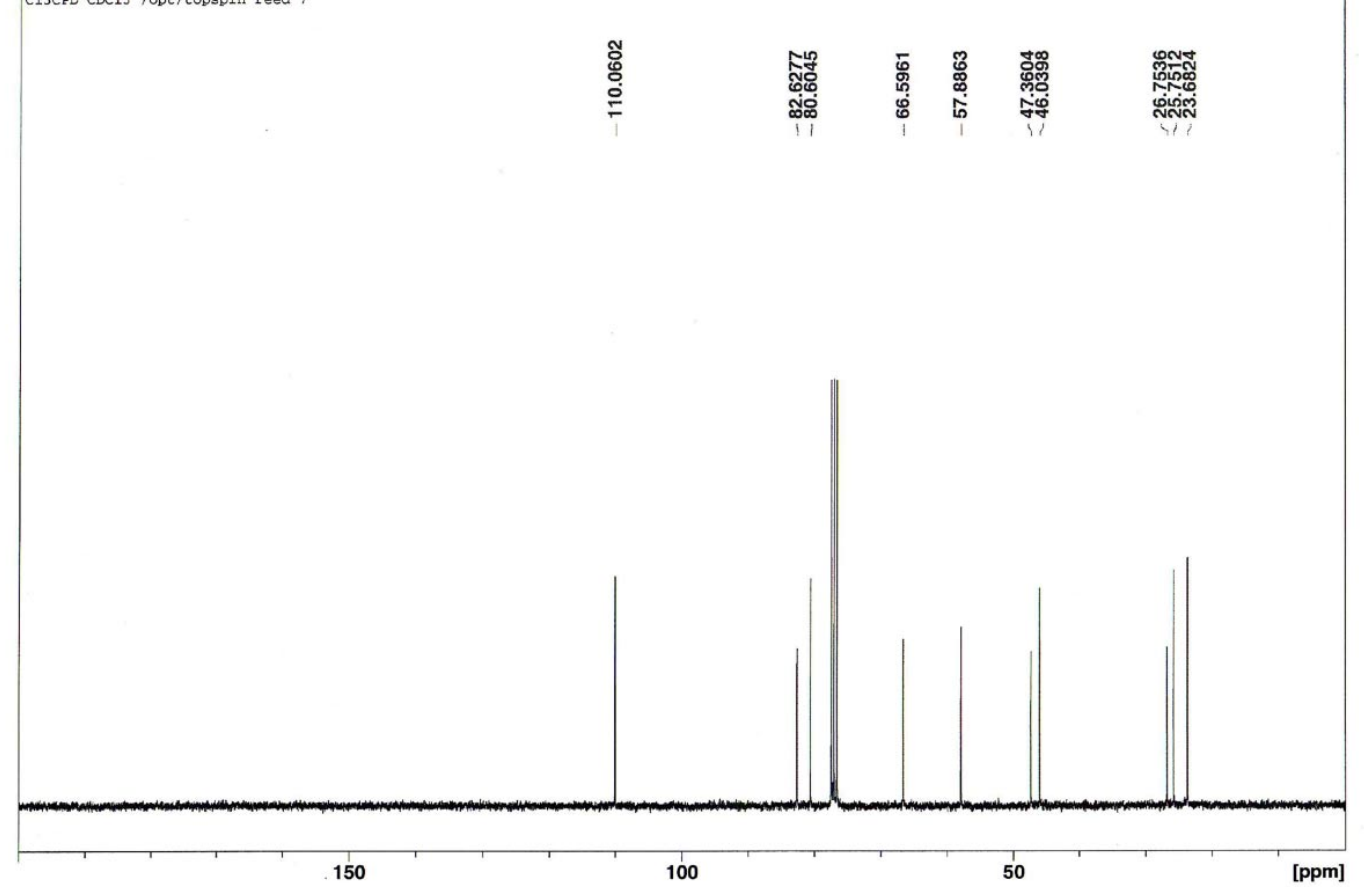


4-exo-hydroxy-6,7-exo-isopropylidenedioxy-N-aza-bicyclo[3.2.1]octane (3)
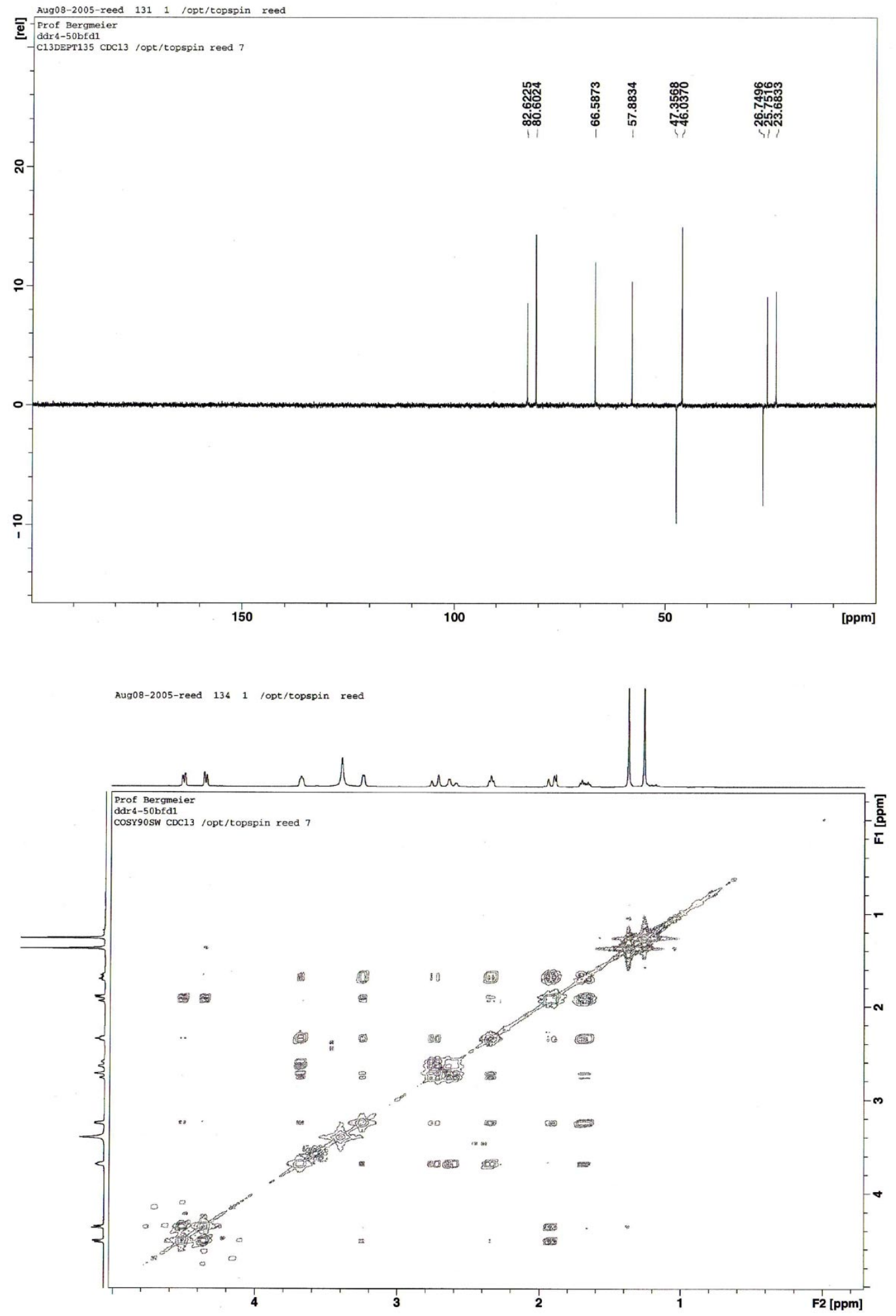